\title{
Impulsive Generation of Coherent Magnons by Linearly Polarized Light in the Easy-Plane Antiferromagnet $\mathrm{FeBO}_{3}$
}

\author{
A. M. Kalashnikova, ${ }^{1,2}$ A. V. Kimel, ${ }^{1}$ R. V. Pisarev, ${ }^{2}$ V. N. Gridnev, ${ }^{2}$ A. Kirilyuk, ${ }^{1}$ and Th. Rasing ${ }^{1}$ \\ ${ }^{1}$ Institute for Molecules and Materials, Radboud University Nijmegen, 6525 ED Nijmegen, The Netherlands \\ ${ }^{2}$ A. F. Ioffe Physico-Technical Institute, Russian Academy of Sciences, 194021 St. Petersburg, Russia
} (Received 2 March 2007; revised manuscript received 27 June 2007; published 19 October 2007)

Polarization-dependent excitation of coherent spin precession by $150 \mathrm{fs}$ linearly polarized laser pulses is observed in the easy-plane antiferromagnet $\mathrm{FeBO}_{3}$. We show that the mechanism of excitation is impulsive stimulated Raman scattering. This process is shown to be determined not only by the magnetooptical constants of the material, but also by the properties of the spin precession itself. Though carrying no angular momentum, the linearly polarized laser pulses act on the spins as effective fields that can be considered as an ultrafast inverse Cotton-Mouton effect.

In recent years, the ultrafast manipulation of the magnetic state of matter by femtosecond laser pulses has emerged as one of the most intriguing issues of magnetism and a subject of tremendous importance for spintronics, information processing, and magnetic recording [1,2]. One of the most important issues in the discussion of the feasibility of all-optical control of magnetization is the possible fast and efficient channels of the angular momentum transfer $[1,3,4]$ from the external stimulus to the spin system and from spins to orbitals and phonons.

Possible scenarios for an ultrafast laser-induced demagnetization and spin switching, based on the simultaneous action of spin-orbit interactions and light, were suggested theoretically in [3]. However, the feasibility of such an alloptical control of spins was repeatedly questioned by others, since the number of photons involved in a conventional experiment is by far not enough to contribute any significant angular momentum [4]. Recently, it was experimentally shown that due to the opto-magnetic inverse Faraday effect, circularly polarized light may excite spin oscillations [5]. The phase of the oscillations was strictly defined by the helicity of light, i.e., by the photon angular momentum. However, the balance of angular momentum remained unclarified, in particular, whether its transfer occurs between light and spins. Therefore, understanding of the actual mechanisms that may provide an effective and ultrafast laser manipulation of spins, revealing the role of the angular momentum and its effective transfer channels, are crucial for optical control of magnetism.

In this Letter, we demonstrate that even linearly polarized $150 \mathrm{fs}$ light pulses that carry no angular momentum can effectively cause coherent spin excitations via impulsive stimulated Raman scattering (ISRS). This mechanism implies the transfer of angular momentum between orbitals and spins mediated by the light and crucially depends on the magnetic structure and orientation of the linear polarization. The reported effect can also be seen as a manifestation of an ultrafast inverse Cotton-Mouton effect where a linearly polarized ultrashort laser pulse acts on spins as a short effective field pulse.

For our study, we chose the iron borate $\mathrm{FeBO}_{3}$ that possesses a good transparency $\left(\alpha \simeq 80 \mathrm{~cm}^{-1}\right.$ at $E=$ $1.54 \mathrm{eV}$ ) and large magneto-optical constants [6-8]. $\mathrm{FeBO}_{3}$ crystallizes in a calcite-type trigonal structure $(\overline{3} m)$ and is an optically uniaxial crystal. The $\mathrm{Fe}^{3+}$ ions occupy two equivalent crystallographic positions forming two sublattices with magnetic moments $\mathbf{M}_{1}$ and $\mathbf{M}_{2}$. They are coupled antiferromagnetically below $T_{N}=348 \mathrm{~K}$ and aligned perpendicular to the $\overline{3}(z)$ axis. The DzyaloshinskyMoriya interaction leads to a canting of the sublattice magnetic moments by an angle of $\simeq 1^{\circ}$ resulting bulk magnetization [9]. The magnetic structure is described by the ferromagnetic vector $\mathbf{M}=\left(\mathbf{M}_{1}+\mathbf{M}_{2}\right)$ and the antiferromagnetic vector $\mathbf{L}=\left(\mathbf{M}_{1}-\mathbf{M}_{2}\right)$. The strong magnetocrysatlline anisotropy is of the easy-plane type. Because of the weak in-plane anisotropy, a magnetic field $H<0.1 \mathrm{kG}$ is sufficient to saturate the sample.

The sample under study was a $d=186 \mu \mathrm{m}$ thick plate with the normal along the optical $z$ axis. The experimental magneto-optical pump-probe setup is described elsewhere [5]. The pump beam with photon energy of $E=1.54 \mathrm{eV}$ and pulse duration of $\Delta \tau=150$ fs was directed along the sample normal. This experimental geometry excludes distortion of the pump polarization by the Faraday rotation and the crystallographic birefringence. The angle of incidence of the probe beam was $10^{\circ}$. Figure 1(a) shows the rotation of the probe polarization induced by the linearly polarized pump pulses as a function of the time delay, for different values of the dc magnetic field applied in the $x y$ plane parallel to the $x$ axis $(\mathbf{M}\|x, \mathbf{L}\| y)$. The frequency of the observed oscillations depends on the magnetic field [Fig. 1(c)], in good agreement with that of the quasiferromagnetic (FMR) mode [Fig. 1(b)]

$$
\Omega_{0}=\gamma \sqrt{H\left(H+H_{D}\right)+2 H_{E} H_{A^{\prime}}}
$$

where $H, H_{D}, H_{E}$, and $H_{A^{\prime}}$ are the applied, Dzyaloshinsky- 


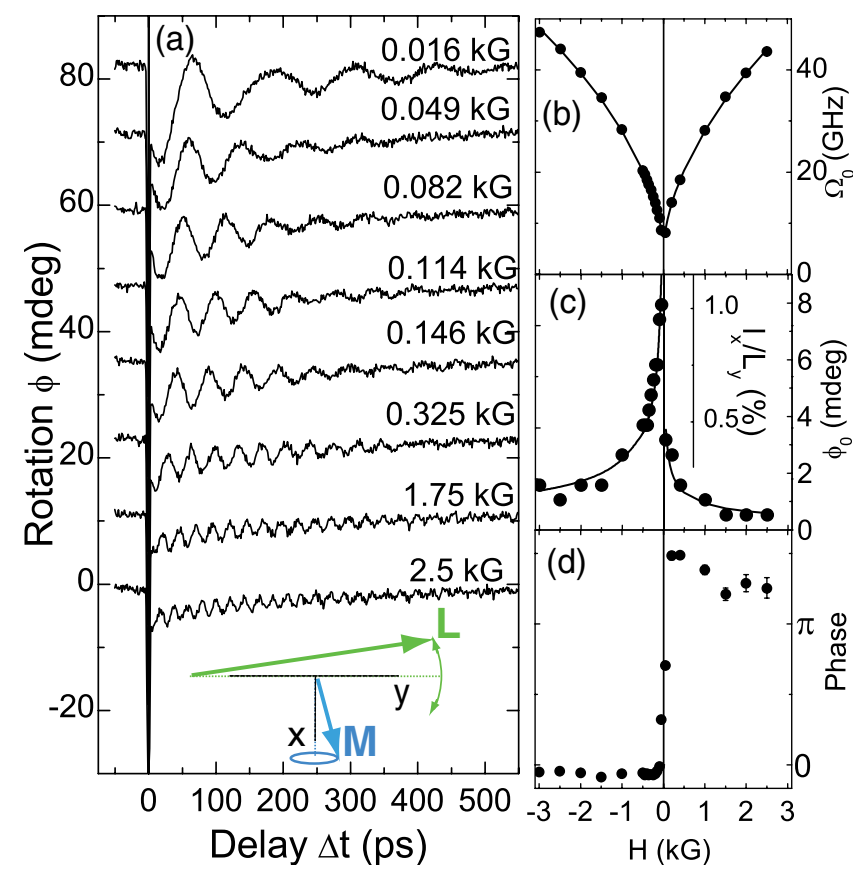

FIG. 1 (color online). (a) The probe polarization rotation vs the time delay between linearly polarized pump and probe pulses for different values of applied magnetic field. (b) Dependence of the oscillation frequency on the magnetic field (symbols) and the field dependence of the FMR mode frequency $\Omega_{0}$ (1). (c) Experimental (symbols) and calculated with Eq. (6a) oscillation amplitude vs the magnetic field. (d) The initial phase of the oscillations vs the applied field.

Moriya, exchange and in-plane anisotropy fields, respectively, and $\gamma$ is the gyromagnetic ratio [9]. We describe the spin precession by deviations $\mathbf{m}(t)$ and $\boldsymbol{l}(t)$ of the ferromagnetic and antiferromagnetic vectors from equilibrium: $\mathbf{M}(t)=\mathbf{M}+\mathbf{m}(t)$ and $\mathbf{L}(t)=\mathbf{L}+\boldsymbol{l}(t)$. In this notation, the FMR mode involves oscillations of the $l_{x}, m_{y}$, and $m_{z}$ components of $\boldsymbol{l}(t)$ and $\mathbf{m}(t)$. The measured rotation of the probe polarization $\phi(t)$ is related to the oscillating components $\mathbf{m}(t)$ and $\boldsymbol{l}(t)$ via the Faraday and magnetic linear birefringence effects. Comparison of the amplitude of the light-induced precession with static magneto-optical measurements shows that the precession angle is $\sim 1^{\circ}$.

Figures 2(a) and 2(d) show the spin precession induced by linearly and circularly polarized pump pulses. In the former case, a clear periodical dependence of the amplitude of the induced oscillations on the pump polarization azimuthal angle $\theta$ is observed [Fig. 2(b)], pointing to a nonthermal mechanism of the excitation. In the latter case, the helicity change appears to affect neither the amplitude nor the phase of the excited precession within the precision of the experiment. This is in contrast to the helicitydependent excitations observed in Ref. [5,10] and described in terms of the inverse Faraday effect. The data in Figs. 2(a) and 2(b) definitely show that it is the linear polarization that controls the excitation process in $\mathrm{FeBO}_{3}$. No spin precession is excited by light polarized parallel or
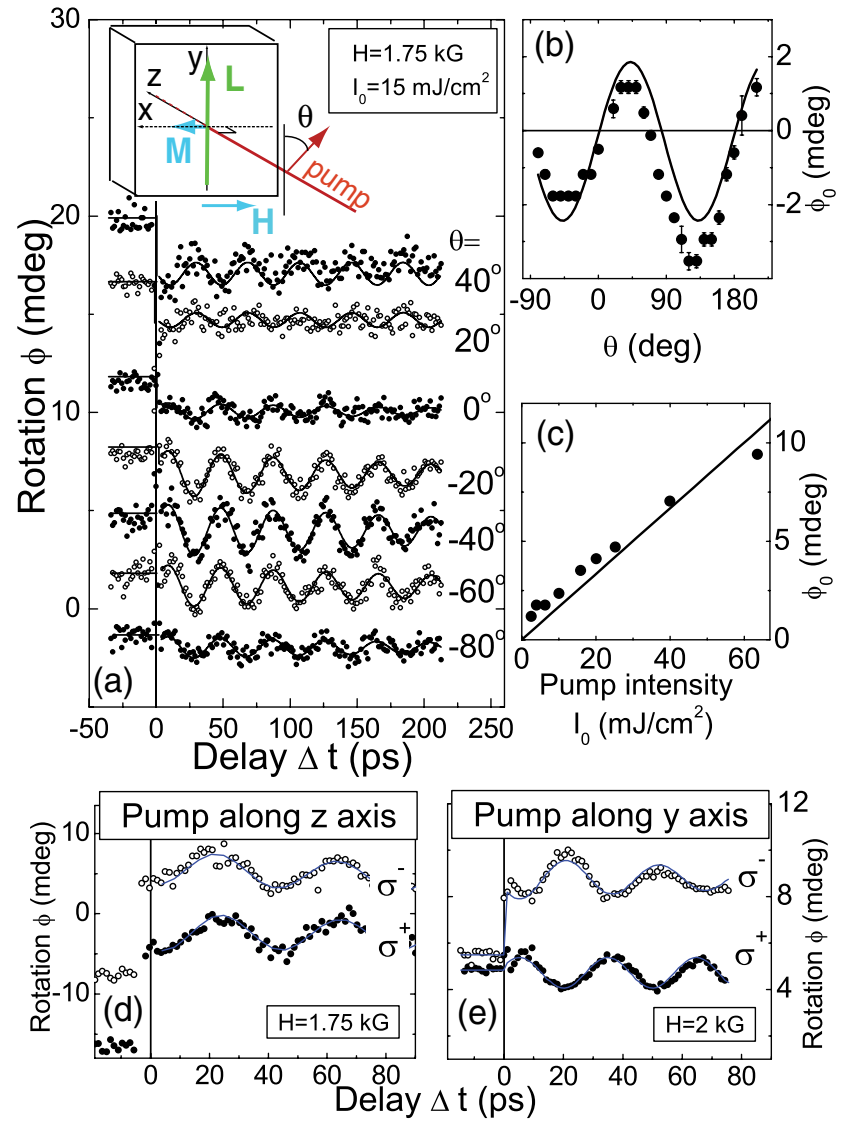

FIG. 2 (color online). (a) The probe polarization rotation vs the time delay between pump and probe pulses for different linear polarizations of the pump (see inset). The oscillation amplitude vs (b) the polarization and (c) intensity of the pump (symbols) and fit with Eq. (6a). (d-e) The rotation of the probe polarization vs the time delay for opposite helicities of the pump pulses propagating along the $z$ and $y$ axes.

perpendicular to the applied magnetic field. We note that the dc-like offsets in Figs. 1(a) and 2(a) possess different field and polarization dependencies than the oscillations themselves and can be ascribed to the light-induced birefringence, but not to perturbations in the magnetic system.

Our data unambiguously show a coherent one-magnon excitation by linearly-polarized light. The excitation mechanism can not be explained in terms of long-living photo-induced anisotropy observed in garnet films [10]. First, the photo-induced anisotropy relies on the absorption by the impurity centers that would lead to saturation of the amplitude of the excited precession at high pump intensity not observed in our experiment [Fig. 2(c)]. Second, an analysis of the spin precession triggered via photo-induced long-living magnetic anisotropy leads to the initial phase of the precession different from the one observed in our experiment [Fig. 1(d)] [11]. Therefore, we should consider an alternative mechanism of the coherent magnon excitation that does not rely on absorption as the photon energy is well below the absorption edge. 
Such a mechanism can be impulsive first order stimulated Raman scattering [12,13], described here in terms of the equation of motion for the normal coordinate $Q(t)$ of the spin wave. The efficiency of such an approach has been demonstrated for the optical excitation of coherent phonons [13-15]. However, due to the time-reversal symmetry breaking in magnetically ordered media, the photonmagnon interactions have distinct features [16]. For the zero wave vector FMR mode, $Q(t)$ is defined through the relation $\left\{l_{x}(t), m_{y}(t), m_{z}(t)\right\}=\operatorname{Re} Q(t)\left\{i a_{x}, i b_{y}, b_{z}\right\}$. Here, the real numbers $\left\{a_{x}, b_{y}, b_{z}\right\}$ define the polarization of the spin wave. For $\mathrm{FeBO}_{3}$, they are [17]

$$
\begin{array}{cc}
a_{x}=\sqrt{2 \gamma M_{0}} \sqrt{\frac{\gamma H_{E}}{\Omega_{0}}}, \quad b_{z}=\frac{1}{2} \sqrt{2 \gamma M_{0}} \sqrt{\frac{\Omega_{0}}{\gamma H_{E}}}, \\
b_{y} / a_{x}=-M_{x} / L_{y}, \quad b_{z} / a_{x}=\Omega_{0} / 2 \gamma H_{E},
\end{array}
$$

where $M_{0}$ is the sublattice magnetization. $Q(t)$ is complex, and in a low-dissipative medium obeys [18]

$$
\frac{d Q(t)}{d t}+i \Omega_{0} Q(t)=-i \frac{\partial \mathcal{H}_{\text {int }}}{\partial Q^{*}} .
$$

The Hamiltonian $\mathcal{H}_{\text {int }}$ describing the interaction of light with the magnetic system has the form

$$
\mathcal{H}_{\mathrm{int}}=-\frac{\delta \varepsilon_{i j}}{16 \pi} \mathcal{E}_{i}(t) \mathcal{E}_{j}^{*}(t)=-\frac{I_{0}}{4 n c} \delta \varepsilon_{i j} e_{i} e_{j}^{*} \delta(t),
$$

where $\delta \varepsilon_{i j}$ is the modulation of the dielectric permittivity by the deviations of the ferromagnetic and antiferromagnetic vectors from equilibrium, $e_{i}, e_{j}$ are the components of the polarization unit vector, $\mathcal{E}_{i}$ and $\mathcal{E}_{j}$ are the time dependent amplitudes of the electric field components of light $\mathbf{E}(t)=\operatorname{Re} \mathcal{E}(t) e^{i \omega t}, n$ is the refractive index, $c$ is the speed of light, and $I_{0}$ is the integrated pulse intensity. The delta function $\delta(t)$ accounts for the impulsive character of the driving force in Eq. (3). Since the frequency of the spin precession $\Omega_{0}$ is much smaller than the central pulse frequency $\omega_{0}$ and the medium is transparent, the dispersion of $\delta \varepsilon_{i j}(\omega)$ was neglected in Eq. (4).

For the light propagating along the $z$ axis, only components $\delta \varepsilon_{i k}$ with $i, k=x, y$ in Eq. (4) are relevant. Using explicit expressions of $\delta \varepsilon_{i k}$ involving components of $\mathbf{m}(t)$ and $\boldsymbol{l}(t)$ [19], it can be shown that $\delta \varepsilon_{x x}$ and $\delta \varepsilon_{y y}$ are responsible for the excitation of the high-frequency antiferromagnetic mode, which is not observed in our experiment. Using the relations $2 l_{x}(t)=i\left(Q-Q^{*}\right) a_{x}$ and $2 m_{z}(t)=\left(Q+Q^{*}\right) b_{z}$, we find the linearized components of the antisymmetric $(a)$ and symmetric $(s)$ parts of $\delta \varepsilon_{x y}$ relevant for the FMR spin precession mode:

$$
\begin{aligned}
& \delta \varepsilon_{x y}^{a}=-\delta \varepsilon_{y x}^{a}=i K m_{z}=\frac{1}{2} i K b_{z}\left(Q+Q^{*}\right), \\
& \delta \varepsilon_{x y}^{s}=\delta \varepsilon_{y x}^{s}=G L_{y} l_{x}=\frac{1}{2} i G L_{y} a_{x}\left(Q-Q^{*}\right) .
\end{aligned}
$$

Here, $K$ and $G$ are the magneto-optical constants defining the magnetic circular and linear birefringence, respectively. Solving (3) using (4) and (5), we obtain expressions for the normal coordinate $Q(t)$ describing the coherent magnons excited by linearly and circularly polarized light. It has been verified experimentally that, primarily due to the strong ellipticity of the precession $\left(b_{z} \ll a_{x}\right)$, the probe polarization rotation in the present geometry is mainly given by the magnetic linear birefringence $\phi(t) \approx$ $\omega_{0} d \delta \varepsilon_{x y}^{s}(t) / n=A G L_{y} a_{x}\left[i\left(Q(t)-Q^{*}(t)\right)\right]$ rather then by the Faraday effect $\phi(t) \sim \delta \varepsilon_{x y}^{a}(t) \sim b_{z}$ which is as small as $\sim 1 \%$ of the former. Here, $A=0.5 \omega_{0} d / n$. Therefore, the rotation of the probe polarization $\phi^{\mathrm{pol}}(t)$ induced by linearly and circularly polarized light is

$$
\begin{aligned}
\phi^{\operatorname{lin}}(t) & =A G L_{y} a_{x}\left[\frac{I_{0}}{4 n c} G L_{y} a_{x} \sin 2 \theta \sin \Omega_{0} t\right], \\
\phi^{\sigma^{ \pm}}(t) & =A G L_{y} a_{x}\left[\mp \frac{I_{0}}{4 n c} K b_{z} \cos \Omega_{0} t\right], \\
\frac{\phi^{\sigma^{ \pm}}}{\phi^{\operatorname{lin}}} & =\mp \frac{K}{G L_{y}} \frac{b_{z}}{a_{x}} .
\end{aligned}
$$

It follows from Eq. (6a) that in $\mathrm{FeBO}_{3}$, spin waves can indeed be excited by linearly polarized light. The efficiency of this process is defined by the magneto-optical susceptibility $G$ and the spin-wave polarization component $a_{x}$. Equations (6) are in nice agreement with the obtained experimental data. Particularly, (i) the polarization dependence of the amplitude of the spin oscillations excited by linearly polarized light [Fig. 2(b)] is in good agreement with Eq. (6a); (ii) the magnetic field dependence of the amplitude of the spin oscillations is well described by Eqs. (6a) and (2): $\phi^{\text {lin }} \sim a_{x}^{2} \sim 1 / \Omega_{0}$; (iii) Equations (6) well account for the fact that in our experiment, the amplitude and phase of the excited spin precession are helicity independent. For $\mathrm{FeBO}_{3}$ at the pump photon energy $1.54 \mathrm{eV}$, the Faraday rotation and magnetic linear birefringence are of the same order of magnitude $K \sim G$. From Eq. (6c), it follows that the ratio between precessions excited by circularly and linearly polarized light is $\sim b_{z} / a_{x}$ and as small as 0.01 [9].

Note that for the light propagating along the $y$ axis, the components $\quad \delta \varepsilon_{x z}^{a}=-\delta \varepsilon_{z x}^{a}=i K_{1} m_{y}+i K_{2} l_{x}=-\frac{1}{2} \times$ $\left(K_{1} b_{y}+K_{2} a_{x}\right)\left(Q-Q^{*}\right)$ of the dielectric permittivity tensor are relevant. Therefore, the helicity-dependent effect of the circularly polarized light $\phi^{\sigma^{ \pm}}(t)$ is defined mainly by the $a_{x}$ component and might be comparable to the effect of the linearly polarized light (6a). Indeed, such an experiment supports this conclusion [Fig. 2(e)].

Thus, even light that does not carry angular momentum is able to excite nonthermally coherent spin precession in a magnetically ordered medium via ISRS. Because of the impulsive character of the stimulated Raman process, direction, polarization and phase of the re-emitted Stokes photon are given by the photon contained in the pump pulse which spectral width exceeds significantly the magnon frequency $\Omega_{0}$ [13]. In ISRS of linearly polarized photons, the Stokes photon remains linearly polarized. It shows that laser-control of spins via ISRS does not require 
angular momentum transfer between photons and spins. Instead, the angular momentum of photons can be conserved, while the simultaneous action of light and spinorbit interaction stimulates the required momentum transfer between the spin and orbital degrees of freedom. We note that the considered process is principally different from the magnon squeezing by linearly polarized short laser pulses reported in [20]. Such a squeezed state was shown to be excited by linearly polarized light via second order ISRS without excitation of coherent magnons.

The ultrafast spin dynamics under the action of the linearly polarized fs laser pulse and the initial phase of the laser-induced spin precession can be described [21] by the Landau-Lifshitz equations [22,23]

$$
\begin{aligned}
\frac{d \mathbf{m}}{d t} & =-\gamma\left(\left[\mathbf{M} \times \mathbf{H}^{\mathrm{eff}}\right]+\left[\mathbf{L} \times \mathbf{h}^{\mathrm{eff}}\right]\right), \\
\frac{d \boldsymbol{l}}{d t} & =-\gamma\left(\left[\mathbf{M} \times \mathbf{h}^{\mathrm{eff}}\right]+\left[\mathbf{L} \times \mathbf{H}^{\mathrm{eff}}\right]\right),
\end{aligned}
$$

where $\mathbf{H}^{\text {eff }}$ and $\mathbf{h}^{\text {eff }}$ are the total effective fields including the fields induced by light. For the two-sublattice $\mathrm{FeBO}_{3}$, one should consider two light-induced effective fields:

$$
\mathbf{H}^{\text {ind }}=-\frac{\partial \mathcal{H}_{\text {int }}}{\partial \mathbf{m}}, \quad \mathbf{h}^{\text {ind }}=-\frac{\partial \mathcal{H}_{\text {int }}}{\partial \boldsymbol{l}},
$$

where the former is known from the theory of optomagnetic interactions for ferromagnetic media [24,25]. The latter accounts for the nonequivalent response of the $\mathrm{Fe}^{3+}$ ions in different crystallographic positions and is specific for multisublattice magnetic media.

For a laser pulse shorter than the period of the spin precession, the effective field induced by a linearly polarized pulse can be written as $h_{x}^{\text {ind }}(t) \propto I_{0} G \sin 2 \theta L_{y} \delta(t)$. Thus, during the action of the laser pulse, the spins experience a torque $d m_{z} / d t=-\gamma L_{y} h_{x}^{\text {ind }}(7)$ and deviate from the equilibrium. Consequently, just after the pump pulse, a $z$ component of $\mathbf{M}$ emerges while $\mathbf{L}$ remains unaffected. The spins begin to precess around the equilibrium directions defined by the internal and applied magnetic fields so that $m_{z}(t) \sim \cos \Omega t$ and $l_{x}(t) \sim \sin \Omega t$. Note that the initial phase of such a precession is in good agreement with our experiment [11]. Therefore, one can see that an ultrashort linearly polarized laser pulse acts on the spins as an effective field pulse. The light-induced effective field $h_{x}^{\text {ind }} \propto$ $G E_{i} E_{j}^{*} L_{y}$ considered above can be seen as an ultrafast nonequilibrium inverse Cotton-Mouton effect $[25,26]$ and is only strong in a magnetically ordered medium due to the large magneto-optical constant $G$ [27,28].

In conclusion, we have shown that linearly polarized 150 fs laser pulses can effectively excite coherent spin precession in a magnetically ordered transparent medium. The microscopic mechanism of the spin precession generation is shown to be impulsive stimulated Raman scattering. The impulsive action of linearly polarized light on a spin system can be seen as an action of an impulsive effective field, and this effect is sizable only in magneti- cally ordered media. The crucial role in the process of optically induced spin precession is played not by the photon angular momentum, but by the magnon properties.

We thank P. A. Usachev for help with the experiments and A.F. van Etteger and A. J. Toonen for technical support. This work is supported by the Dutch Network NanoNed, RFBR, NWO, INTAS, the program Spintronics of RAS, and FASI.

[1] J. Stöhr and H. C. Siegmann, Magnetism. From Fundamentals to Nanoscale Dynamics (Springer-Verlag, BerlinHeidelberg, 2006).

[2] I. Zŭtić et al., Rev. Mod. Phys. 76, 323 (2004).

[3] R. Gómez-Abal et al., Phys. Rev. Lett. 92, 227402 (2004).

[4] B. Koopmans et al., Phys. Rev. Lett. 85, 844 (2000).

[5] A. V. Kimel et al., Nature (London) 435, 655 (2005).

[6] I. Bernal et al., Acta Crystallogr. 16, 849 (1963).

[7] Landoldt-Börnstein Numerical Data and Functional Relationships, edited by K.-H. Hellwege, New Series, Group III, Vol. 27h (Springer-Verlag, Berlin, 1993).

[8] A. J. Kurtzig et al., Appl. Phys. Lett. 14, 350 (1969).

[9] J. Schober, IEEE Trans. Magn. 12, 401 (1976).

[10] F. Hansteen et al., Phys. Rev. Lett. 95, 047402 (2005).

[11] Solving the LL-equations for $\mathrm{FeBO}_{3}$, one can see that the spin precession induced via photo-induced anisotropy is described by $l_{x}(t) \sim(1-\cos \Omega t)$ with an initial phase of the motion that is in disagreement with our data.

[12] Y. R. Shen and N. Bloembergen, Phys. Rev. 143, 372 (1966).

[13] Y. Yan et al., J. Chem. Phys. 83, 5391 (1985).

[14] R. Merlin, Solid State Commun. 102, 207 (1997).

[15] T. Deskorsy, G. C. Cho, and H. Kurz, in Light Scattering in Solids VIII, edited by M. Cardona and G. Güntherodt (Springer, Berlin, 2000).

[16] R. Loudon, J. Raman Spectrosc. 7, 10 (1978).

[17] V. S. Lutovinov and V. L. Safonov, Sov. Phys. Solid State 22, 1541 (1980).

[18] V.E. Zakharov and E. A. Kuznetsov, in Soviet Scientific Reviews, edited by S. P. Novikov, Mathematical Physics Reviews (OPA, Amsterdam, 1984), Sect. C.

[19] E. A. Turov, J. Exp. Theor. Phys. 85, 797 (1997).

[20] J. Zhao et al., Phys. Rev. Lett. 93, 107203 (2004).

[21] This more traditional approach to the laser-induced spin dynamics supplements the rigorous one based on ISRS.

[22] L. D. Landau and E. M. Lifshitz, Statistical Physics, Part 2 (Pergamon Press, New York, 1980), Part 2.

[23] A. S. Borovik-Romanov and N. M. Kreı̌nes, Phys. Rep. 81, 351 (1982).

[24] P. S. Pershan, Phys. Rev. 130, 919 (1963).

[25] P. S. Pershan et al., Phys. Rev. 143, 574 (1966).

[26] The term inverse Cotton-Mouton effect was earlier introduced without specifying the medium, although inverse Voigt effect would be more appropriate for the magnetic media where this effect is understood as a light-induced effective field rather then change of a magnetization.

[27] G. A. Smolenskiı̌ et al., Sov. Phys. Usp. 18, 410 (1975).

[28] J. Ferré and G. A. Gehring, Rep. Prog. Phys. 47, 513 (1984). 